\title{
Ägyptische Analogien zum Funde des Deuteronomiums.
}

Von Privatdozent Lic. Johs. Herrmann, Wi en.

In einem Aufsatze in den Proceedings of the Society of Biblical Archaeology vom Dezember 1907 (S. 232-242), betitelt „Ägyptische Schriftstücke in Grundmauern und das Alter des Buches Dt" brachte NAVILLE verschiedenes ägyptisches Material zur Illustrierung von 2 Reg 228 vor. Der Aufsatz erregte vielfaches Interesse wohl vor allem wegen der Art, in welcher NAviLLE den vorgebrachten Stoff verwertete. Insbesondere in OLZ erschien eine Reihe kleiner Artikel, durch Navilles Abhandlung hervorgerufen (vgl. Hubert GrIMME in OLZ io, Sp. 6i I, Dez. Ig07; W. ERBT OLZ II, Sp. 57-62, Febr. 1908; P. HAUpt ebenda Sp. II9 bis 125, März 1908; E. KöNIG ebenda Sp. 125-127). Auf dieselben soll hier nicht eingegangen werden; das Material NAVILLEs soll vielmehr einer kurzen Besprechung und Beurteilung unterzogen werden. Doch ist NAville nicht der einzige und nicht der erste, der ägyptischen Stoff zur Illustrierung von 2 Reg 228 bemerkt und mitgeteilt hat. CHEYNE erinnerte kürzlich (in OLZ II, Sp. 195. April 1908) daran, daß kein Geringerer als MASPERO der erste war, ${ }^{x}$ da $\$$ seine Anregung aber als erster Biblicalscholar CHEYNE selbst aufgegriffen hat, und zwar in seinem Jeremiah, his life and times (S. 85) 1888. Letztere Stelle hat nachher MARTI in ZThK 1892 S. 44 erwähnt (in einem Aufsatze „Das erste offizielle Bekenntnis“). In Navilles Aufsatz ist die Mitteilung zweier Inschriften aus Dendera am bedeutsamsten, auf die NAville zum ersten Male hinweist. Es handelt sich dabei entschieden um einen ähnlichen Vorgang, wie der in 2 Reg 228 erzählte es ist. In diesem Lichte erscheint auch KITTEL die Sache, der sie kürzlich in seinem Vortrage "Die orientalischen Ausgrabungen und die ältere biblische Geschichte"s 1908 (S. $42 \mathrm{f}$.) streifte, wobei er mit Recht bemerkte: „Die Frage, wie es bei der Herstellung und Auffindung des im 2. Königsbuch erwähnten Mosegesetzes hergegangen sei, ist natürlich damit nicht ohne weiteres erledigt." Trotzdem bleibt die

21878 in Revue critique, dann auch in Hist. anc. de l'Orient 73. 
Parallele beachtenswert genug. Ihr möge zunächst unsere Aufmerksamkeit gelten.

Die zwei Stellen aus den Inschriften des großen Hathortempels von Dendera finden sich beide in Krypte 9, die eine an der Südwand, die andere an der Ostwand. Veröffentlicht wurden sie in DúmicheNs „Bauurkunde der Tempelanlagen von Denderah" von 1865, in desselben Verfassers "Baugeschichte des Denderahtempels" von 1877 und in MARIETTEs großer Publikation der Denderainschriften, die $1870-74$ in 4 Bänden erschien (A. MARIETTE, Denderah. Description générale du grand temple). Für diesen Artikel stellte mir HermanN JUNKER in Wien seine Abschrift und Übersetzung freundlichst zur Verügung, wofür ich ihm auch hier herzlich danke.

Ich gebe zunächst die beiden Stellen in Urschrift, Umschrift und Übersetzung und bezeichne sie dann weiterhin nur mit A (an der Südwand) und B (an der Ostwand).

A. Dendera, Krypte 9, Südwand (cf. MarietTe, Dendera; III $78 \mathrm{n}$. DüMICHEN, Bauurkunde, XV. DümICHEN, Baugeschichte 1.):

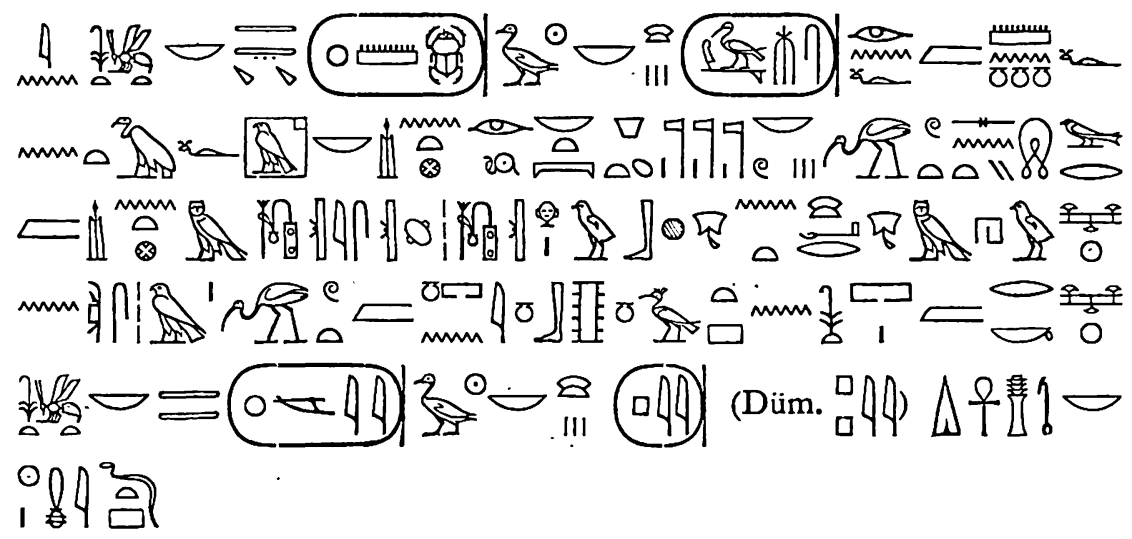

\section{Umschrift:}

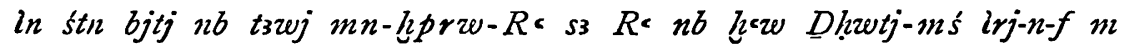
mnw.f $n$ mwt.f Ht-lir $n b$.t In.t ir.t $R \times n b . t p$.t hnze.t ntr.wnb.w

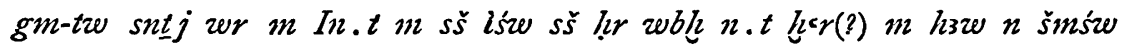
Hr gin tw(?) m lnww inb $n$ db.t $n$ pr śtn $m$ rk śtn bjtj nb tзzej mrj

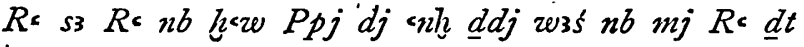

Übersetzung: Der König von Ober- und Unterägypten, der Herr der beiden Länder Mn hprw R`, der Sohn des R`, der Herr der Diademe, Thutmosis machte (es) als sein Denkmal für seine Mutter Hathor Herrin 
von Dendera, die den Rc hervorbrachte, Herrin des Himmels, Herrin aller Götter. Gefunden wurde der große Plan von Dendera-in alter Schrift, geschrieben auf ein ... fell, aus der Zeit der Horusdiener. Gefunden wurde er (?) im Innern einer Ziegelmauer des Königshauses aus der Zeit des (p. t.) Mrj R c (p. t.) Ppj (der beschenkt ist mit Leben etc.).

B. Dendera, Krypte 9, Ostwand (cf. Mariette III 78 k. Dưmichen, Bauurkunde, XVI. DÜMICHEN Baugeschichte I.):

(Unter der Darstellung:)
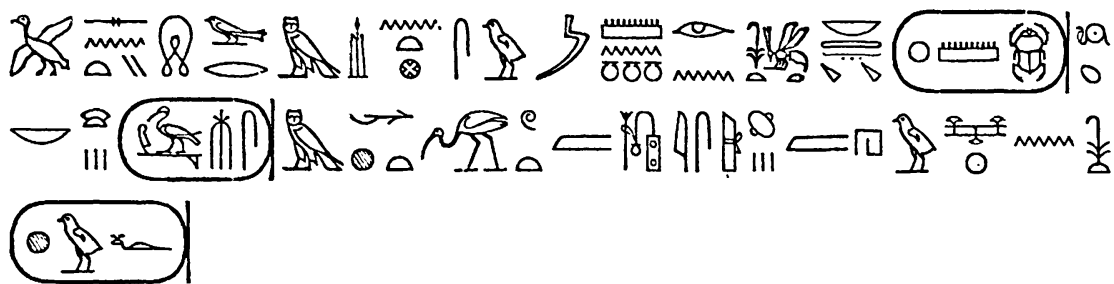

Umschrift:

(Unter der Darstellung:)

p3 sntj wr m In.t Sinzwj mnw irj $n$ śtn bjtj nb tзwj $m n-h p r w-R \times s 3$

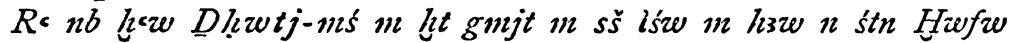

Úbersetzung: Der große Plan von Dendera. Erneuerung des Denkmales, welches gemacht hatte der König (p. t.) Mn-hprw-Rc, nachdem man ihn gefunden in alter Schrift aus der Zeit des Königs Chufu.

2 Reg 22 3-10 erfahren wir, wie dem Kanzler Safan, der in offizieller Mission im Tempel weilt, vom Oberpriester Hilkia ein Buch übergeben wurde mit den Worten: „Ein Gesetzbuch habe ich im Hause Jahwes gefunden." Die Sendung des Safan bestand darin, den Geldsammelkasten, dessen Inhalt für Reparaturarbeiten verwendet wurde, zu leeren und die Gelder den mit der Aufsicht über diese Arbeiten Betrauten zu übergeben. Die Ankündigung und Übergabe des Fundes kommt sehr unvermittelt, so daß KrTTEL eine Lücke vor v. 8 , wahrscheinlich fand. Unwillkürlich ist man geneigt, zwischen den Zeilen zu lesen, daß die Auffindung vielleicht irgendwie mit den Reparaturarbeiten im Tempel zusammengehangen habe. Die Vermutung wird allerdings durch keine Aussage im Text gestützt. Aber bei der Kürze und verhältnismäßigen Dunkelheit des ganzen Abschritts mul schon etwas Kombinieren gestattet sein. Warum wird der Zweck des Zusammentreffens von Kanzler und Oberpriester so hervorgehoben? Unser Text läbt den Oberpriester dem Kanzler nur die Tatsache des Buchfundes mitteilen. Aber findet man ein Gesetzbuch auf 
der Straße? Es kann nur dort gefunden werden, wo es aufbewahrt worden ist. Also entweder bei einer Revision des Tempelarchivs, oder bei Reparaturarbeiten im Tempel. Letzteres erscheint mir nicht unwahrscheinlich. Damit ist übrigens die Frage noch gar nicht berührt, ob etwa der Oberpriesțer eine Mystifikation vornahm oder ob er das Buch wirklich gefunden hat, sondern nur dies, daß er es dem Kanzler als bei einer Revision des Tempelarchivs bezw., was man vielleicht $z$ wischen den Zeilen lesen darf, bei Reparaturarbeiten im Tempel an verborgenem Ort, in einem Mauerhohlraum oder sonstwo gefunden präsentierte.

Die beiden Dendera-Stellen berichten ebenfalls von dem Fund einer Urkunde. Gefunden wurde der große śnt $\mathrm{j}$ von Dendera. $s \mathrm{~s}_{\mathrm{t}} \mathrm{j}^{\mathrm{x}}$ heißt Grund, Boden, Fundament, Grundriß, Plan, auch Art und Weise. Was heibt das Wort in unserm Falle? Wir müssen den Zusammenhang der beiden Stellen einzeln prüfen. A steht am Ende einer die Südwand von Krypte 9 ausfüllenden Liste. Dieselbe beginnt mit dem Namen von Dendera, zählt die Götter von Dendera mit ihren Namen auf, gibt die Lage ihrer Gemächer an, nennt Feste, die den Göttern gefeiert werden. Am Schlusse der Liste, die einen etwas krausen und wirren Eindruck macht, folgt der Kalender der Hathorfeste, schließend mit der Prozession der Göttin nach Edfu am I. Mesore. Dann kommt unsere Stelle.

B steht am Anfang einer Liste, welche gleichfalls mit dem Namen von Dendera beginnt. Daran schließen sich die Namen des Heiligtums und der Halle von Hathor Horus und $i$ h j (also 6 Namen) und 9 Priesternamen. Auf der Westwand steht: Namen der Sängerinnen, der heiligen Stätten, der heiligen Bäume, der heiligen Schlangen, der heiligen Gewässer. Aus der Vergleichung mit ähnlichen Listen in Edfu, auf die mich JUNKER aufmerksam macht (siehe ROCHEMONTEIX, le temple d'Edfou, I, 339, 358f.), darf man schließen, daß die Angaben auf der Westwand die Fortsetzung der Liste auf der Ostwand bilden; soviel ich sehen kann, ${ }^{2}$ erlauben das auch die lokalen Verhältnisse der Krypte 9. Es ist nun deutlich, daß diese Liste mit śntjw $\mathbf{r}$ bezeichnet ist. Dann ist aber auch in $A$ anzunehmen, da $\$$ śnt $\mathrm{j}$ keinesfalls etwa blo $ß$ auf das Fest am I. Mesore gehen, sondern gleichfalls die ganze Liste bezeichnen wird. Oder noch mehr? Beide Listen, die vor $A$ und die nach $B$, sehen nicht wie Teile einer großen Liste, sondern wie zwei selbständige Ganze aus. Dann hätten wir also zwei große Pläne von Dendera, die gefunden worden sind?

× Vg]. auch Dưmichen, Bauurkunde, S. 19.

2 Dümichens Baugeschichte ist mir leider nicht zur Hand. 
B will, wie mir scheint, sagen, dieser große Plan, der folgen soll, sei eine Erneuerung des Denkmals, welches Thutmosis III gemacht hatte, der das Denkmal aber auch nicht neu erfand, sondern es nach einem, nämlich eben diesem, großen Plane machte, der gefunden worden war aus der Zeit des Königs Chufu (ca. 2900). Es unterliegt nun keinem Zweifel, was mit dem „Denkmal“ gemeint ist; der Denderatempel der Ptolemäerzeit ist eine genaue Nachbildung des Tempels des Thutmosis III, welcher seinerseits nach unserer Stelle die Tempelanlage auf Grund alter Schriften, gefunden, aus der Zeit des Chufu stammend, gemacht haben soll. Es wird in unserer Stelle so geredet, als sei die Liste nach B einfach der große Plan von Dendera. In Wirklichkeit gehört zu dem natürlich noch vieles andere, wie uns die Liste von $A$ zeigt. Ist aber aus $B$ deutlich, was mit dem „Denkmal“. gemeint ist, so muß auch in A sintjwr den großen Plan der Tempelanlage von Dendera bezeichnen, also auf die ganze vor A stehende Liste sich beziehen. Auch hier wird von dem s $n t j w r$ berichtet, daß er in alten Schriften gefunden wurde. Aber nicht aus der Zeit des Chufu sondern aus der Zeit der Horusdiener, d. i. der vordynastischen Könige stammend, und zwar im Innern einer Ziegelmauer des Königshauses aus der Zeit des Pepj (ca. 2600), geschrieben auf ein Tierfell.

Wer bis hierher unserm Deutungsversuch wohlwollend gefolgt ist, sieht, da@ wir vor gewissen Schwierigkeiten stehen. Die Frage ist: wollen unsere beiden Stellen sagen, da $\$$ eben diese Liste vor $A$ bzw. nach $B$ nach ihrem Wortlaute der śn $\mathrm{tj} \mathbf{j} \mathrm{r}$ von Dendera sei, der aus alter Zeit stammend, aufgefunden, von Thutmosis III zu seinem Neubau von Dendera verwendet worden sei? Der Ton liegt dem Schreiber offenbar darauf, nachzuweisen, da $b$ die $z u$ seiner Zeit bestehende Tempelanlage eine genaue Nachbildung derjenigen des Thutmosis sei, und dab auch dessen Werk nur Erneuerung auf Grund noch älterer Pläne war. Plan bezeichnet aber hier, wie die Listen zeigen, nicht bloß die lokalen Verhältnisse, sondern auch andere Einrichtungen des Tempels: Benennungen, Feste etc. Ist in $A$ von dem Funde der Liste vor $A$, in $B$ von dem der Liste nach $B$ die Rede, so haben wir zwei verschiedene Funde aus verschiedenen Zeiten. Thutmosis hätte, nach Denderaischer Überlieferung, seinerzeit einen gefundenen Plan aus der Zeit der Horusdiener, sowie einen solchen aus der des Chufu benützt. Das wäre an sich wohl möglich. Allein die Duplizität des Falles erscheint doch etwas bedenklich, ganz abgesehen von dem oben über die Art der beiden Listen Bemerkten. Ich möchte mir darum eine Vermutung erlauben, die einen andern Sachverhalt ergibt. Vielleicht liegt die Sache etwas einfacher. Wie wir z. B. aus den oben angeführten 
Listen in Edfu sehen, war es nichts einzigartiges, Listen von Tempeleinrichtungen an die Wände der Tempel zu malen. Im Tempelarchiv befanden sich verschiedene, die dem Maler als Vorlage dienen konnten. $\mathrm{Da}$ nun die Tradition ging, daß Thutmosis die Anlage von Dendera auf Grund eines gefundenen ś $n \underline{t} j$ aus alter Zeit gemacht habe, so wurden solche Pläne dementsprechend bezeichnet. Die Nachrichten über die Zeit, aus der der śnt $\mathrm{j} w \mathbf{r}$ des Thutmosis stammte, gingen auseinander. Der eine von den in Krypte 9 mitgeteilten soll aus der Zeit des Chufu, der andere gar aus der Zeit der vordynastischen Könige stammen. Die Tendenz solcher Nachrichten ist deutlich, sie erklärt die $Z$ wiespältigkeit der Überlieferung; nach den einen stammte der śnt $\mathbf{j}$ aus der Zeit eines historischen Königs - vielleicht war dies wirklich irgendwie historisch begründet - nach den andern aber gar aus sagengrauer Vorzeit. Der wirklich gefundene śnt $\mathrm{t}$, den Thutmosis verwendet hatte, war nicht mehr vorhanden. Nun konnte man verschiedene Listen von tatsächlichen Tempeleinrichtungen mit gutem Gewissen als ś $\mathrm{t} j \mathrm{j}$ r von Dendera gleich dem des Thutmosis bezeichnen, weil man ja wußte, daß er die ganze Tempelanlage nach dem gefundenen $\sin \hat{t} j$ vorgenommen. Wie man schwerlich jede Teilliste an sich als śntjwr bezeichnen konnte, so hat auch Thutmosis nur einen śn $\underline{\mathrm{t} j \mathrm{w}} \mathrm{r}$ benützt, nicht zwei aus verschiedenen Zeiten stammende Funde. Dagegen hat sich dann die Überlieferung gespalten und es werden zwei in verschiedener Art angelegte Pläne als der śntjwr bezeichnet, den Thutmosis gefunden hatte. Es dürfte demnach $\mathrm{m}$. E. nur von einem Urkundenfund die Rede sein, und die verschiedene Altersangabe dürfte auf Verschiedenheit der Überlieferung über die gleiche Sache zurückgehen.

Beide Stellen enthalten demnach die Nachricht, daß Thutmosis III bei seiner Erneuerung der Tempelanlagen von Dendera sich eines aus alter Zeit stammenden Planes bediente, der in einer Ziegelmauer des Königshauses aus der Zeit. des Pepj gefunden worden war. Niemand wird leugnen, daß dies wirklich der Fall gewesen sein kann. $O b$ es so war, unterliegt nun freilich den gleichen Bedenken, wie der Bericht von dem Fund des Gesetzbuches 2 Reg 22. Denn wenn man in letzterem bestimmte Tendenzen wahrnimmt, welche die verwirklichen wollten, die sie vertraten, so kann auch in der Nachricht von Dendera eine Tendenz liegen, nämlich die Tempeleinrichtungen von Dendera als aus sehr alter Zeit stammend, nicht erst aus der des Thutmosis, hinzustellen, vielmehr letzteren nur als Erneuerer auszugeben. Andererseits aber ist von vornherein ebenso sicher, daß sehr wohl das ganze Denderawerk des Thut- 
mosis nichts anderes als die Erneuerung des in alter Urkunde Überlieferten gewesen sein kann. Auch betreffs des Berichts in 2 Reg 22 muß man sagen, då sehr wohl ein vor der Manasseschen Reaktion verfaßtes Gesetzbuch, welches die Tendenzen der hiskianischen Reformzeit verwirklichen wollte, nach Eintreten der Reaktion absichtlich im Tempel, sei es im Archiv, sei es sonstwo, verborgen und in der Zeit Josias wieder aufgefunden worden sein kann.

Damit scheint über die Bedeutung der Denderanachricht schon genug gesagt. Sie berichtet uns von dem Fund einer sakralen Urkunde, die als Grundlage des Werkes Thutmosis' III in Dendera dienen soll, welches eben dadurch als Erneuerung uralter Überlieferung erscheint. Auch das Gesetzbuch, das Hilkia dem Safan überreicht, erscheint durch die Mitteilung, daß es gefunden worden sei, als eine Urkunde der Vergangenheit. In beiden Fällen ist die Historizität der Nachricht an sich recht wohl möglich. In beiden Fällen kann der Verdacht entstehen, daß die Nachricht von dem Funde dem Interesse daran ihren Ursprung verdankt. In beiden Fällen reicht dieser Verdacht schwerlich aus, die Glaubwürdigkeit der Nachricht zu vernichten. Die Gründe für dieselbe sind in jedem der beiden Fälle nach Lage der Sache verschieden. Die Denderanachricht ist uns also von großem Interesse, weil sie von einem Falle erzählt, der in gewisser Hinsicht Ähnlichkeit mit dem in 2 Reg 22 vorliegenden hat. Die Glaubwürdigkeit des letzteren Berichtes wird dadurch zwar nicht über allen Zweifel erhoben, erhält aber eine nicht unwesentliche Stütze, indem wir nun von einem ähnlichen Vorgang an anderem Orte erfahren; die, welche den Fund in 2 Reg 22 schon an sich so schwer glaublich fanden, werden. ihn vielleicht nun etwas glaublicher finden müssen. Die Glaublichkeit der Denderanachricht ist entschieden recht groß. Auf die Restauration des alten Tempels zielt nach Dumichen, Bauurkunde S. I9 auch hin der (auf Tafel V unter Nr. 6 dort verzeichnete) Name von Dendera die größte Wahrscheinlichkeit für sich, daß wirklich das Werk Thutmosis' in Dendera im engsten Anschluß an alte Überlieferung geschah. Wenn irgendwo, so waren in Ägypten die alten Überlieferungen hoch angesehen und mabgebend. Noch in der Ptolemäerzeit erstand eine Periode, in der man allenthalben die alte Zeit wieder lebendig machen wollte. Aber schon in alter Zeit ging man gern und womöglich auf die Überlieferung zurück. Das Werk des Ketzerkönigs konnte deshalb in Ägypten nur von ganz vorübergehender Wirkung sein. Von Amenem ha êct I (II. 
Dynastie), der die Grenzen der Städte wieder herstellte, wird in der Inschrift des Chnem hôtep in Benihassan (LEPSIUS, Denkmäler II r24; ERMaN, Ägypten, 68, 135) rühmend hervorgehoben, dal er sich über die Wassergebiete der einzelnen Städte aus dem unterrichtete, was in den Büchern stand, und sie nach dem verzeichnete, was in alten Schriften stand, weil er die Wahrheit so sehr liebte. Das ist nur ein Beispiel. Waren in der Hyksoszeit allerorts die Fäden der alten Úberlieferung abgerissen worden, so war es Sache der Könige der 18. Dynastie, die Fäden von neuem zu knüpfen, und jedermann weiß von den großartigen Erneuerungsbauten dieser Könige, insonderheit auch des Thutmosis III.

Wir wollen allerdings nicht vergessen, daß der Vorgang in der Denderainschrift und in 2 Reg 228.zwar gewisse Ähnlichkeiten bietet, die wir möglichst hell ans Licht stellten, daß es aber dabei sein Bewenden haben muß. Insonderheit ist und bleibt, wenn unsre Auffassung der Denderainschrift richtig ist, der $s \mathrm{n} t \mathrm{jwr}$ von Dendera etwas anderes als das Gesetzbuch in 2 Reg 22, mag immerhin sowohl in der Inschrift von

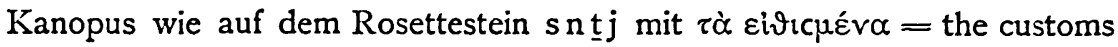
übersetzt sein, wie NAviLle mitteilt. In Dendera ist $\mathrm{s} n \underline{\mathrm{t}} \mathrm{j}$ vom Grundrib oder Plan der Tempellokalitäten aus auf die damit verbundene Mitteilung von kultischen Einrichtungen (dazu gehören auch die Kultpersonen) ausgedehnt, die mehr oder weniger mit den Tempellokalitäten zusammenhangen.

Der $\operatorname{sinjw} r$ wird in einer Ziegelmauer aus der Regierungszeit des $P_{\mathrm{Pj}}$ gefunden. Thutmosis erneuert in Dendera, wie Naville vermutet, in Stein, was er in Ziegeln gefunden hat, wie in Theben und an anderen Orten. NAville weist nun darauf hin, daß eine so alte Mauer zu den Grundmauern gehört haben müsse, und damit ist ihm der Fall ein Beleg der. Gewohnheit der alten Ägypter, Bücher in die Grundmauern zu legen, welche an ihrem verborgenen Platz für Jahrhunderte aufgehoben sein konnten, bis sie gelegentlich bei Renovations- oder Restaurationsarbeiten gefunden wurden. In dem Bericht 2 Reg 22 steht nichts davon, daß das Gesetzbuch in den Grundmauern des salomonischen Tempels gefunden wird. Es dürfte methodisch unzulässig sein, aus der von NAville bei den Ägyptern beobachteten Gewohnheit zu schließen, auch der Fund des Gesetzbuchs, von dem Hilkia dem Safan redet, müsse als in den Tempelgrundmauern geschehen gedacht werden. Wären wir aber, was nicht der Fall ist, aus jenem oder irgendeinem Anlasse gezwungen, das Wort. Hilkias in der eben bezeichneten Weise aufzufassen, und ist es uns aus andern Gründen gewi $₫$, daß das unter Josia gefundene Gesetzbuch irgendwie in dem 5. Buch das Pentateuch enthalten ist, so folgte nicht, daß 
Hilkias Buch das in die Grundmauer des salomonischen Tempels eingemauerte Gesetzbuch wäre, sondern es wäre dann nur sicher, daß die Behauptung Hilkias eine Fiktion war. Denn eine absolute Garantie dafür, daß Hilkia wirklich das Gesetzbuch gefunden hat, wie er sagt, besitzen wir nicht.

Wir haben bis jetzi die Denderainschriften isoliert behandelt. Es gibt aber noch andere ägyptische Beispiele von Funden alter Schriftstücke. Wir wollen zunächst NAvILLEs weiteres Material betrachten. Es handelt sich erstens um eine Beischrift zum 64. Kapitel des Totenbuchs, die in der ältesten Abschrift dieses Kapitels sich findet (abgeschrieben durch WiLkINSON S. GoodwiN in $\ddot{A}$. Ztschr. I866 S. 53) und heruntergeht bis in die Zeiten der Ptolemäer und Römer in den spätesten Abschriften, von denen eine große Anzahl erhalten ist. Sie lautet z. B. Totenbuch ed. NAville II Pl. 99: „Dieses Kapitel wurde gefunden in Šmun auf einem Ziegel oder einer Platte oder einem Stein des Südens unter den Füßen Sr. M. des ehrwürdigen Gottes in der Schrift des Gottes selbst zur Zeit Sr. M. des Königs von Ober- und Unterägypten Menkaura. Der Königssohn Hordudef fand es, als er auf dem Wege war, die Tempel von Ägypten zu inspizieren (inspect)." In andern Texten folgen darauf noch Worte, von welchen NAviLLE sagt, daß er sie nicht sicher angeben kann, welche ihm aber anzuzeigen scheinen, daß dem Königssohn irgend jemand das Geschriebene erklärte, so daß er verstehen konnte, wie wertvoll es war. „Und er (der Königssohn) brachte es zum König als ein Wunder, als er sah, daß es etwas Geheimnisvolles war, was niemand gesehen oder betrachtet hatte." Mit Recht weist NAVILLE die Ansicht zurück, es handle sich hier um eine Art Betrug, durch welche die Priester diesem Kapitel einen sehr alten Ursprung beilegten, um es verehrungswürdiger zu machèn. Denn wie käme gerade dieses Kapitel bzw. diese Gruppe von Kapiteln zu solcher Ehre, die es, allein mit einem andern teilt, von dem noch zu sprechen sein wird? Der Inhalt der Beischrift ist, wie NAville feststellt, dab in sehr, alter Zeit dieses Kapitel, auf eine Steinplatte graviert, unter der Statue des Thot deponiert worden ist und zwar in dem Tempel, welcher besonders ihm geweiht war, nämlich im Tempel zu Šmun. Von dieser Tatsache aus schließt Naville auf die Sitte, ein Deposit unter der Statue des Thot zu machen und vielleicht auch unter den Statuen anderer Götter. Da Thot vor allem Gott der Schrift und der Bücher ist, so mag man unter die Basis seiner Statue eines der Bücher gelegt haben, von welchen man annahm, dab es seine Worte seien. Einen weiteren Beleg der Sitte sieht NAville darin, daß 
Hogartir im Tempel von Ephesus unter dem Platze, wo die Statue der Artemis stand, einen Schatz von Goldornamenten fand, der zur Zeit der frühesten Tempelanlage an diese Stelle gelegt zu sein scheint. Man mag darüber und über das weitere, was NAviLLE zu der Sache bemerkt, denken wie man will: Tatsache ist jedenfalls, da 6 von Kap. 64 des Totenbuches bzw. einer Gruppe von Kapiteln erzählt wird, es sei als Steininschrift unter den Füßen des Thot im Tempel von Šmun gefunden worden, und daß diese Behauptung so fest an dem betreffenden Kapitel haftet, wie eine ganze Anzahl Texte aus verschiedenen Zeiten zeigen, dab man sie schwerlich für eine Fiktion halten darf. Die betreffende Partie des Totenbuchs muß aber jedenfalls in besonderem Ansehen gestanden haben, weshalb man sie unter die Füße des Thot gelegt haben wird.

Dafür spricht auch die merkwürdige Tatsache, die NAVILLE weiterhin mitteilt. Es existiert nämlich von Kap. 64 noch eine kurze Version, welche die Überschrift trägt: „Kapitel um kennen zu lernen die Kapitel des Ausgangs aus dem Tage in einem Kapitel" (Totenb. II Pl. 132 BuDGE, the book of the Dead, Text S. 142). Gegenuiber der längeren Form von Kap. 64, von der oben die Rede war, mit der Überschrift „Kapitel vom Ausgang aus dem Tage" haben wir hier eine zusammengedrängte Form mehrerer Kapitel vom Ausgang aus dem Tage. Dabei findet sich die Beischrift: „Dieses Kapitel wurde gefunden in den Grundmauern von Amihunun durch den Aufseher der Männer, welche die Mauer bauten, zur Zeit des Königs Usaphais; seine Figuren sind geheimnisvoll, niemand hat sie gesehen oder auf sie geblickt." Was die Zeitangabe anlangt, so möchte ich vermuten, daß sie sich nicht, wie Naville meint, auf die Zeit der Entdeckung bezieht, sondern daß zu übersetzen ist „welche bauten die Mauer aus der Zeit des Usaphais“, das heißt, welche die aus der Zeit des Usaphais stammende Mauer renovierten; doch habe ich den Text nicht vor mir. Naville sieht hier ein Beispiel der Gewohnheit vor sich, Schriftstücke in den Grundmauern der Tempel niederzulegen. Wie ausgedehnt diese Gewohnheit war, wissen wir nicht. Jedermann sieht die Ähnlichkeit der Notiz mit den Angaben über den Fund des s $n \underline{t} \mathbf{j} w \mathbf{r}$ von Dendera. Eine Nötigung, dieselbe für eine Fiktion $z \mathrm{u}$ halten, liegt, wie mir scheint, nicht vor. Steht aber hinter der Notiz ein wirklicher Vorgang, so haben wir, wie in Dendera, auch hier den . Fund eines Schriftstückes in einer alten Mauer, und wenn die Niederlegung dieses Totenbuchtextes auch nicht. so naheliegend erscheint, wie die des sntjwr von Dendera, so mag sie sich doch daraus erklären, $\mathrm{da} ß$ der Inhalt jenes Textes besonders wichtig und verehrungswürdig er- 
schien. Es braucht wohl nicht besonders darauf hingewiesen zu werden, daß beide Fälle neben Ähnlichkeiten doch auch beträchtliche Unterschiede aufiveisen.

CHEyne erwähnt an der im Eingange dieses Aufsatzes bezeichneten Stelle außer der Beischrift bei der längeren Fassung von Kap. 64 des Totenbuchs, die NAVILLE in mehrfachen Versionen mitteilt, noch weitere zwei Bücherfundberichte. Erstens findet sich bei einem Kapitel eines medizinischen Papyrus im Brit. Museum folgende Rubrik (in Abschrift mitgeteilt von BIRCH in $\ddot{A g}$. Ztschr. IS7I S. 63): "Dieses Nittel wurde entdeckt in der Nacht durch die Hand eines Tempeldieners der Göttin, welcher zufällig in der Halle des Tempels der Stadt Tebmut im Geheimgemach dieser Göttin war. Das Land war zu dieser Zeit in Dunkel gehüllt, aber der Mond schien voll auf das Buch. Es wurde als ein wertvoller Schatz zu Sr. M. dem König Cheops gebracht." In einer Berliner medizinischen Abhandlung, die BRUGSCH herausgegeben hat, heilft es: „Gefunden wurde sie in alter Schrift in einer Buchlade zu Füßen des Gottes Anup von Sekhem in den Tagen der Heiligkeit des Königs von Oberund Unterägypten des Wahrhaftigen" (vgl. BRUGSCH, Gesch. Ägyptens" S. 60; Maspero, Hist. anc. de l'Orient ${ }^{2}$ S. 57). Ich gebe die bei Cheyne mitgeteilte Übersetzung wieder; doch habe ich die Texte selbst nicht gesehen. Die erste der beiden Rubriken zeigt Märchenstil. Die zweite gibt nüchterne Angaben und zeigt gewisse Berührungen mit der Beischrift zu Kap. 64 des Totenbuchs; vielleicht äußert sich NAvILLE einmal näher dazu. Ich wüßte nicht, warum sie nicht von einem wirklichen Fund berichten sollte. Cheyne meint, es sei eine konventionelle Fiktion der Priester gewesen, zu sagen, daß ein Buch in einem Tempel gefunden sei, um die Autorität des Buches zu stützen. Das ist möglich, beweist aber für die einzelnen Fälle nichts, und die Anzahl derselben scheint auch zu gering. Sollte es aber eine Stilform gewesen sein, Schriftstücke gelegentlich als in einem Tempel gefunden fingierenderweise $z u$ bezeichnen, so würde sich diese Stilform am ehesten daraus erklären, daß es in der Tat nichts Außergewöhnliches war, wenn' hier und da ein altes Schriftstück gefunden wurde, sei es nun in Tempelmauern, sei es. vielleicht auch in Tempelarchiven.

Eine interessante Stelle, die ich bei ERMaN, Ägypten, S. 480 erwähnt fand, möchte ich zum Schlusse noch mitteilen. Im Papyrus Ebers steht bei einem Arzneimittel $(75,12)$ die Beischrift: ,Siehe das ist ein wirkliches Mittel. Es ist bei einer Revision im Tempel des Wennofre gefunden." Auch diese Stelle illustriert das eben Gesagte. 
Von allem hier vermerkten Material sind für den Alttestamentler ohne Zweifel die beiden Denderainschriften am wichtigsten. Doch verdienen, neben ihnen und mit ihnen, auch all die andern mehr oder weniger analogen Fundberichte von Schriftstücken unsere Aufmerksamkeit. Gewiß werden die Kenner ägyptischen Schrifttums noch weiteres Material beibringen. Mag dann der oder jener Fundbericht aus im einzelnen Falle vorliegenden Gründen nur als eine Stilform mit bestimmter Tendenz erscheinen, so ist uns, wie vorhin bemerkt, doch die Entstehung jener Stilform interessant. In jedem Falle aber haben wir für alles Material dankbar zu sein, welches irgendwie 2 Reg 228 illustriert. Was insbesondere die Bedeutung der Denderainschriften nach dieser Richtung hin anlangt, so habe ich oben versucht, sie.mit Vorsicht und Zurückhaltung abzuschätzen. Der Wert von Analogien bleibt immer ein relativer. Über das andere Material habe ich im wesentlichen nur referiert. Auf keilinschriftliche Stellen einzugehen, die zur Erläuterung des Deuteronomiumfundes herangezogen worden sind (vgl. H. WINCKLER in. OLZ 1900 Sp. 376 und $\mathrm{KAT}^{3}$ 1903 S. 277; A. JEREMIAS $\mathrm{ATAO}^{2} 1906 \mathrm{~S}$. 548), war nicht Gegenstand des vorliegenden Aufsatzes. 